\title{
Intraepithelial and lamina propria lymphocytes show distinct patterns of apoptosis whereas both populations are active in Fas based cytotoxicity in coeliac disease
}

\author{
A Di Sabatino, R Ciccocioppo, S D’Alò, R Parroni, D Millimaggi, M G Cifone, \\ G R Corazza
}

\begin{abstract}
Background-Lamina propria (LPLs) and intraepithelial (IELs) lymphocytes are markedly increased in coeliac mucosa, and are thought to play a crucial role in the generation of villous atrophy in coeliac disease (CD). However, the mechanisms by which they mediate the killing of enterocytes in this condition are still poorly characterised.
\end{abstract}

Aim-We investigated Fas mediated cytotoxicity and apoptosis of both LPLs and IELs, isolated from 10 untreated coeliac patients, 10 coeliac patients on a gluten free diet, and 10 biopsied controls.

Methods-Fas and Fas ligand expression were assessed by flow cytometry and immunocytochemistry. Lymphocyte cytotoxicity against Fas expressing Jurkat cells was determined by the Jam test. The effect of the antagonist ZB4 anti-Fas antibody on apoptotic activity exerted by coeliac lymphocytes against enterocytes was analysed. Lymphocyte apoptosis was assessed by oligonucleosome ELISA.

Results-LPLs and IELs showed increased apoptotic activity and higher levels of Fas ligand expression in untreated CD compared with treated CD patients and controls. Enterocyte apoptosis observed after coculturing coeliac lymphocytes and enterocytes in the presence of ZB4 antibody was reduced. In active CD, LPLs manifested increased apoptosis whereas IELs showed decreased apoptosis.

Conclusions-Our results support the involvement of the Fas/Fas ligand system in CD associated enterocyte apoptosis. Increased LPL apoptosis is likely to downregulate mucosal inflammation whereas decreased IEL apoptosis could be responsible for autoimmune and malignant complications of $\mathrm{CD}$.

(Gut 2001;49:380-386)

Keywords: apoptosis; coeliac disease; cytotoxicity assay; Fas/Fas ligand system; intraepithelial lymphocytes; lamina propria lymphocytes

Professor G R Corazza, Unità di Gastroenterologia, IRCCS Policlinico San Matteo, Piazzale Golgi 5 , 27100 Pavia, Italy. gr.corazza@smatteo.pv.it

Accepted for publication 12 February 2001 Coeliac disease (CD) is a $\mathrm{T}$ cell mediated enteropathy characterised by villous atrophy suggested to be due to increased enterocyte apoptosis, ${ }^{12}$ and by a high density of lamina propria (LPLs) and intraepithelial (IELs) lymphocytes. $^{3}$ Activated T LPLs are markedly increased in coeliac mucosa $a^{4}$ and their crucial role in inducing mucosal flattening is well recognised. ${ }^{5}$ IELs have been shown to mediate Fas ligand (FasL) cytotoxicity against Fas expressing enterocytes in a murine model of graft versus host disease, ${ }^{67}$ a condition which shares many pathological and immunological similarities with CD. Moreover, in a recent study ${ }^{8}$ we have shown that in untreated CD, increased IEL FasL expression correlated significantly with the level of enterocyte apoptosis.

Thus to demonstrate effective involvement of LPLs and IELs in determining increased enterocyte apoptosis in CD, we evaluated the Fas/FasL cytotoxic mechanism ${ }^{9}$ in this condition. In addition, as lymphocyte apoptosis may contribute to limit expansion of activated $\mathrm{T}$ cells, ${ }^{10}$ and to delete autoreactive $\mathrm{T}$ cell clones, ${ }^{11}$ we also investigated LPL and IEL apoptosis, as dysregulation of programmed cell death of these cells may favour the autoimmune and malignant complications of CD.

\section{Patients and methods}

PATIENTS AND TISSUES

Size appropriate and well oriented endoscopic biopsy specimens (12-15 per patient) were obtained from the second part of the duodenum in 10 untreated coeliac patients (mean age 37.3 years, range 23-61), 10 coeliac patients on a gluten free diet (mean age 34.8 years, range 20-65) for at least 12 months, and 10 consenting subjects (mean age 39.2 years, range 28-62) undergoing upper gastrointestinal endoscopy for functional dyspepsia. Subjects using steroidal or non-steroidal antiinflammatory drugs or presenting with an inflamed mucosa at histology were excluded from the study. Some of the biopsy samples were processed according to standard methods for traditional histology and others were used to obtain purified LPL, IEL, and enterocyte suspensions.

Abbreviations used in this paper: $\mathrm{CD}$, coeliac disease; FasL, Fas ligand; IELs, intraepithelial lymphocytes; LPLs, lamina propria lymphocytes; PBS, phosphate buffered saline; FCS, fetal calf serum; TUNEL, terminal deoxynucleotidyl transferase mediated digoxigenin-deoxyuridine triphosphate nick end labelling. 
CELL ISOLATION

Biopsy specimens were collected in sterile medium (phosphate buffered saline (PBS) calcium and magnesium free, supplemented with $100 \mathrm{U} / \mathrm{ml}$ penicillin, $100 \mu \mathrm{g} / \mathrm{ml}$ streptomycin, and $5 \%$ fetal calf serum (FCS)). The epithelial layer was removed with $1 \mathrm{mM}$ ethylenediamine-tetraacetic acid (EDTA; Sigma Chemical Co, St Louis, Missouri, USA) and $1 \mathrm{mM}$ dithiothreitol (Sigma). After continuous agitation for one hour at $37^{\circ} \mathrm{C}$, the single cell suspension was pelleted from the supernatant and washed once with $5 \mathrm{ml}$ of RPMI 1640 medium (Gibco, Life Technologies Ltd, Paisley, UK) supplemented with antibiotics and 10\% FCS. IELs and enterocytes were then separated on a Percoll density gradient (Pharmacia Fine Chemicals, Pharmacia, Inc., Uppsala, Sweden). A discontinuous density gradient $(25 \%, 40 \%$, and $75 \%)$ was used. Cells that layered between the $40 \%$ and $75 \%$ fractions were collected as IELs whereas cells that layered between the $40 \%$ and $25 \%$ interface were collected as enterocytes. To release lamina propria mononuclear cells, the remaining tissue was washed three times with RPMI 1640 medium and treated with type 1A collagenase $128 \mathrm{U} / \mathrm{ml}$ (Sigma) for three hours with continuous agitation at $37^{\circ} \mathrm{C}$. The crude cell suspension was allowed to stand for five minutes to permit sedimentation of debris. Cells from the supernatant were washed twice with RPMI 1640 medium containing 10\% FCS, $100 \mathrm{U} / \mathrm{ml}$ penicillin, and $100 \mu \mathrm{g} / \mathrm{ml}$ streptomycin, and layered on Ficoll-Hypaque solution (Pharmacia) to isolate LPLs by a density gradient method. Single cell suspensions were resuspended in $1 \mathrm{ml}$ of PBS and kept on ice until used. The viability of single cell suspensions was determined by trypan blue exclusion. Cells were not used if viability did not exceed $90 \%$. The purity of enterocyte and IEL populations, determined by processing cell cytocentrifugates with anticytokeratin pan antibody at 1:400 dilution (Boehringer Mannheim, Indianapolis, Indiana, USA), exceeded $95 \%$ and $80 \%$, respectively. The purity of the LPL population, assessed by flow cytometric analysis on a FACS II analyser (FACScan; Becton Dickinson Co., San Jose, California, USA), was $90 \%$. Purified enterocytes as targets and both LPLs and IELs as effectors were used in the cytotoxicity assay.

IMMUNOFLUORESCENCE AND FLOW CYTOMETRY Freshly isolated LPLs were washed twice in PBS containing $2 \% \mathrm{FCS}$ and incubated at $4{ }^{\circ} \mathrm{C}$ for 30 minutes with phycoerythrine labelled anti-Fas mouse MoAb IgG1 (Coulter/ Immunotech, Inc., Westbrook, Minnesota, USA) and fluorescein isothiocyanate labelled anti-FasL mouse MoAb IgG2 (Upstate Biotechnology, Lake Placid, New York, USA). Based on forward and side scatter, cells were gated on the area corresponding to lymphocytes and analysed by a FACScan (Becton Dickinson). Fluorescein isothiocyanate labelled IgG1 and phycoerythrine labelled IgG2 (Becton Dickinson) were used as control reagents to exclude non-specific binding and to determine the optimal setting of fluorescence quadrants.

IMMUNOCYTOCHEMISTRY

Isolated IELs were placed on electrostatic slides by means of cytocentrifugation, fixed by incubation for 10 minutes in cold acetone/ methanol 1:1, and air dried. Afterwards, cells were incubated for five minutes in $3 \%$ hydrogen peroxide to quench endogenous peroxidase activity, rinsed in PBS, and incubated with bovine serum albumin $2 \%$ in PBS for 30 minutes. Subsequently IELs were incubated for 60 minutes at room temperature with the antihuman FasL IgG1 mouse monoclonal antibody (clone NOK-1; Pharmingen, San Diego, California, USA) at 1:150 dilution. Cells were rinsed in PBS and treated with a secondary biotinylated antibody and peroxidase conjugated streptavidin. Finally, diaminobenzidine reaction and nuclear counterstaining with Harris' haematoxylin were performed. As a positive control, the TM4 cell line (immortalised mouse Sertoli cells) was used. As a negative control, slides of IELs were processed without the primary antibody. Sections were examined using conventional light microscopy in a blinded fashion by an expert observer. Counts were performed at a constant magnification $(\times 1000)$ by a differential count of at least 500 cells and the results expressed as mean percentage.

JAM TEST

To investigate whether FasL expressed on LPLs and IELs was functional, we analysed the ability of LPLs and IELs to kill Fas expressing Jurkat cells. DNA fragmentation in $\mathrm{Fas}^{+}$target cells (human $\mathrm{T}$ cell leukaemia, Jurkat) after four hours of coculture with LPLs or IELs was performed as previously described. ${ }^{12}$ Briefly, Jurkat cells $\left(2 \times 10^{4}\right)$ were pulsed for 24 hours with $2 \mu \mathrm{Ci} / \mathrm{ml}$ of ${ }^{3} \mathrm{H}$ thymidine $(6.7 \mathrm{Ci} / \mathrm{mmol}$; NEN, Boston, Massachusetts, USA), washed, and then incubated either alone or with effector cells at different effector/target ratios (5:1, 2.5:1, 1.25:1, and 0.625:1 for LPLs; and 3:1, $1.5: 1$, and $0.625: 1$ for IELs). At the end of the culture, cells were harvested on a glass fibre filter, and ${ }^{3} \mathrm{H}$ thymidine present on the filters was measured by liquid scintillation counting. The results are expressed as mean (SD) cpm of five replicate cultures. The percentage of fragmented DNA was calculated as follows: $100 \times($ mean cpm target cells cultured alone-mean cpm effector cells cultured with target cells/mean cpm target cells cultured alone).

\section{CYTOTOXICITY ASSAY}

To determine if enterocyte apoptosis in CD involves interaction between Fas receptor expressed on target cells (enterocytes) and FasL expressed on effector cells (LPLs or IELs), the effect of an antagonist ZB4 anti-Fas antibody (Ancell Corporation, Bayport, Minnesota, USA) was analysed. LPLs and IELs (effector cells) and enterocytes (target cells), isolated from both untreated coeliac patients and biopsied controls, were incubated at $37^{\circ} \mathrm{C}$ 


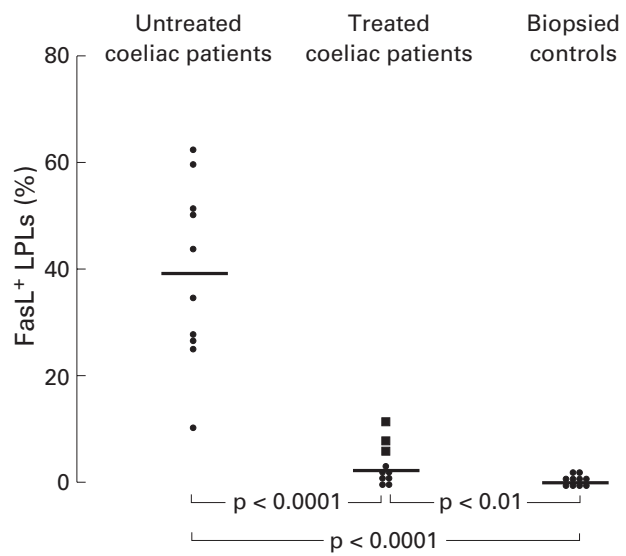

Figure 1 Percentages of FasL $L^{+}$lamina propria lymphocytes (LPLs) in 10 untreated coeliac patients compared with those of 10 treated coeliac patients and 10 biopsied controls. Those coeliac patients on a gluten free diet and with a persistent degree of villous atrophy are indicated with a different symbol (solid square). Horizontal bars represent median values.

in a $5 \% \mathrm{CO}_{2}$ atmosphere for four hours in the absence or presence of $2 \mu \mathrm{g} / \mathrm{ml} \mathrm{ZB} 4$ anti-Fas blocking added to the culture medium. The effector/target ratios were 100:1 in experiments involving LPLs and 20:1 in those involving IELs. Aliquots of both untreated coeliac and biopsied control enterocytes were cultured without effector cells to give an estimation of spontaneous enterocyte apoptosis either in the absence or presence of ZB4. Afterwards, cocultured cells were placed on electrostatic slides by means of cytocentrifugation, fixed by incubation for 10 minutes in paraformaldehyde $3.7 \%$, air dried, and permeated by means of acetone/methanol 1:1. Finally, cells were processed for terminal deoxynucleotidyl transferase mediated digoxigenin-deoxyuridine triphosphate nick end labelling (TUNEL) detection using the peroxidase ApopTag Kit (Oncor, Gaithesburg, Maryland, USA) and observed under light microscopy to evaluate the percentage of apoptotic enterocytes by a differential count of at least 300 cells at a constant magnification $(\times 1000)$.

OLIGONUCLEOSOME ELISA

Qualitative and quantitative in vitro determination of cytoplasmic histone associated DNA fragments (mono and oligonucleosomes) from LPLs and IELs were performed with the Cell Death Detection ELISA (Boehringer Mannheim).

\section{MUCOSAL MORPHOMETRY}

To quantify the persistence of mucosal abnormality in treated $C D$, we measured the surface area to volume ratio $(\mathrm{S} / \mathrm{V})$ of duodenal mucosa. $\mathrm{S} / \mathrm{V}$ in biopsy specimens was estimated by a Weibel graticule (Graticules Ltd, Tunbridge Wells, UK) inserted in the microscope eyepiece, according to the method of Dunnil and Whitehead. ${ }^{13}$ A record was made of the number of times the lines cut the mucosal surface (c) and the number of hits (h) - that is, end points of the lines falling on the lamina propria. At a constant magnification $(\times 125)$ the length $(1)$ of each line of the graticule was $1.7 \times 10^{-2} \mathrm{~cm}$. The

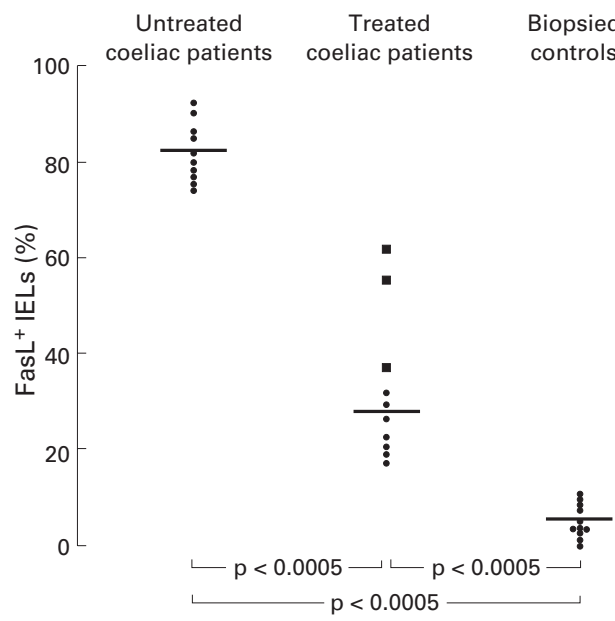

Figure 2 Percentages of Fas $\mathrm{L}^{+}$intraepithelial lymphocytes (IELs) in 10 untreated coeliac patients compared with those of 10 treated coeliac patients and 10 biopsied controls. Those coeliac patients on a gluten free diet and with a persistent degree of villous atrophy are indicated with a different symbol (solid square). Horizontal bars represent median values.

ratio $\mathrm{c} / \mathrm{lh}$ was then calculated and regarded as a measure of $\mathrm{S} / \mathrm{V}$.

Those treated patients with an $\mathrm{S} / \mathrm{V}$ ratio lower than 57 were considered to have some persistent degree of mucosal abnormality, according to our previous study. ${ }^{14}$

\section{STATISTICAL ANALYSIS}

Data are expressed as median (range). Statistical comparisons were carried out using the Mann-Whitney U test for non-parametric data. A level of $p<0.05$ was considered statistically significant.

\section{Results}

FasL EXPRESSION BY LPLS AND IELs

Flow cytometry was used to analyse expression of FasL on the plasma membrane of LPLs. Otherwise, as the number of viable isolated IELs (mean $1.5 \times 10^{6}$, range $0.5-2.3$ ) was insufficient to perform all of the experiments, immunocytochemistry instead of flow cytometric analysis was employed to evaluate FasL expression by these cells.

Figure 1 shows flow cytometric analysis of FasL expression by LPLs isolated from biopsy specimens obtained from untreated coeliac patients, treated coeliac patients, and biopsied controls. In untreated CD, the percentage of $\mathrm{FasL}^{+}$LPLs (median 39.3\%, range 10.3-61.4) was significantly higher compared with treated CD (median 2.6\%, range 0.2-10.7) and controls (median $0.5 \%$, range $0.1-1.7$ ). In treated CD, the percentage of $\mathrm{FasL}^{+}$LPLs was still significantly higher than in controls.

Figure 2 shows that in untreated CD almost all IELs were $\mathrm{FasL}^{+}$and their percentage (median $80.8 \%$, range 75.4-92.3) was significantly higher than in treated patients (median $27.5 \%$, range 18.2-63.5) and controls (median $5.8 \%$, range 1.2-10.9). In treated $\mathrm{CD}, \mathrm{FasL}^{+}$ IELs were still significantly higher than in controls.

After treatment, levels of FasL expressed by both LPLs and IELs were higher in those three patients with persistent villous atrophy. 

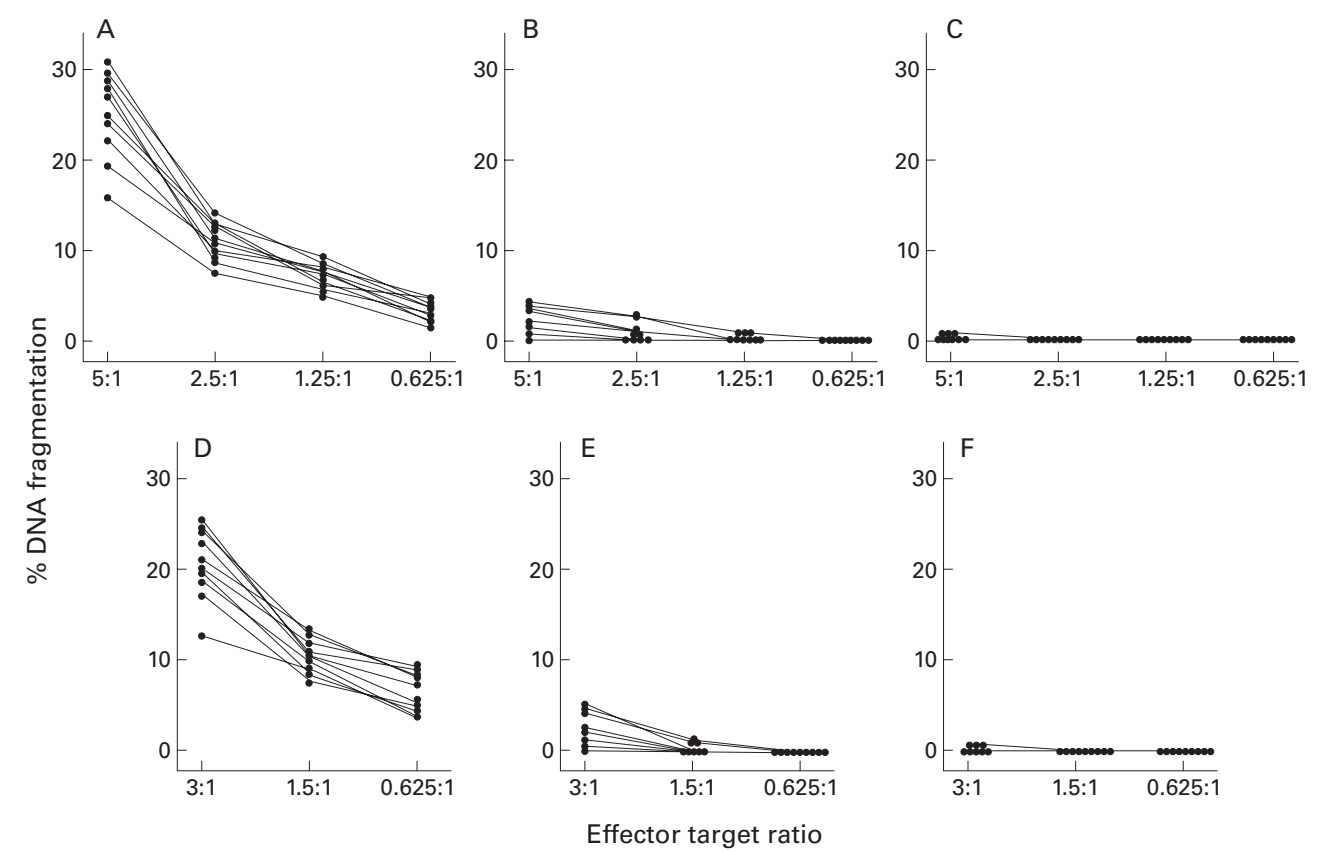

Figure 3 FasL mediated cytotoxicity by lamina propria lymphocytes (LPLs) (effectors) obtained from 10 untreated coeliac patients $(A), 10$ treated coeliac patients $(B)$, and 10 biopsied controls $(C)$ against Furkat cells (targets). ${ }^{3} \mathrm{H}$ labelled furkat cells were cultured with LPLs at various effector/target ratios $(5: 1 ; 2.5: 1 ; 1.25: 1 ;$ and $0.625: 1)$. The percentage of DNA fragmentation of Jurkat cells was assayed after 12 hours. FasL mediated cytotoxicity by intraepithelial lymphocytes (IELs) (effectors) obtained from 10 untreated coeliac patients (D), 10 treated coeliac patients (E), and 10 biopsied controls (F) against furkat cells (targets). ${ }^{3} \mathrm{H}$ labelled furkat cells were cultured with IELs at various effector/target ratios (3:1; 1.5:1; and 0.625:1). The percentage of DNA fragmentation of Jurkat cells was assayed after 12 hours.

Untreated coeliac LPLs + enterocytes

Untreated coeliac IELs + enterocytes

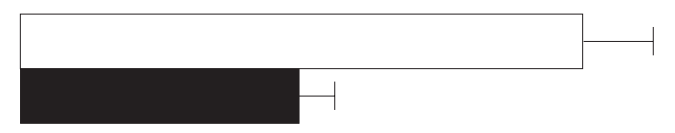

Untreated coeliac enterocytes

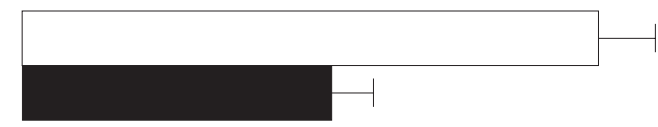

Biopsied control LPLs + enterocytes

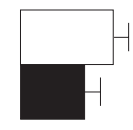

Biopsied control IELs + enterocytes

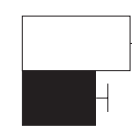

Biopsied control enterocytes

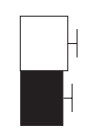

$$
0
$$

20

\section{0 \\ $\%$ Apoptosis}

Figure 4 Antagonist ZB4 anti-Fas antibody reduces enterocyte apoptosis induced by both lamina propria lymphocyte (LPL) and intraepithelial lymphocyte (IEL) cytotoxic activity through a Fas/FasL interaction. Untreated coeliac and control enterocytes alone, and coculture of both untreated coeliac and control LPLs and IELs with enterocytes (at an effector target ratio of 20:1 and 100:1, respectively) were incubated for four hours in the presence or absence of $2 \mu \mathrm{g} / \mathrm{ml} \mathrm{ZB4}$ antagonist antibody. The percentage of apoptotic cells was determined by TUNEL detection.
JAM TEST

Figure 3 shows the killing activity of LPLs freshly isolated from untreated coeliac mucosa (fig 3A), treated coeliac mucosa (3B), and normal mucosa (3C). In untreated CD (3A), this activity was related to the effector target ratio and remained evident at the lowest ratio of $0.625: 1$. At each ratio this killing activity was significantly higher $(\mathrm{p}<0.0005)$ than those observed when LPLs from treated (3B) or normal mucosa (3C) were used. In treated CD, LPL cytotoxicity was significantly higher than in normal mucosa only at the highest effector target ratio.

Regarding the cytotoxicity of IELs, at each ratio their killing activity was significantly higher $(p<0.001)$ using IELs isolated from untreated coeliac mucosa (3D) than that using IELs from treated (3E) or normal mucosa (3F). Similar to LPLs, a gluten free diet, which also led to a clear improvement in duodenal histology, did not normalise cytotoxicity of IELs.

\section{CYTOTOXICITY ASSAY}

To confirm that the Fas/FasL cognate interaction was the mechanism by which LPLs and IELs mediated the killing of enterocytes in CD, the antagonist ZB4 anti-Fas antibody which blocks the Fas/FasL interaction was added to the coculture of coeliac lymphocytes and enterocytes. After four hours of coculture, most target untreated coeliac enterocytes when incubated with untreated coeliac LPLs or IELs were $\mathrm{TUNEL}^{+}$(68.4 (8.1)\% and $67.4(9.3 \%)$, respectively). In contrast, when $\mathrm{ZB} 4$ anti-Fas blocking was added to the coculture, both percentages significantly decreased (36.4 (5.1)\% and 32.8 (4.8)\%, respectively), as shown in fig 

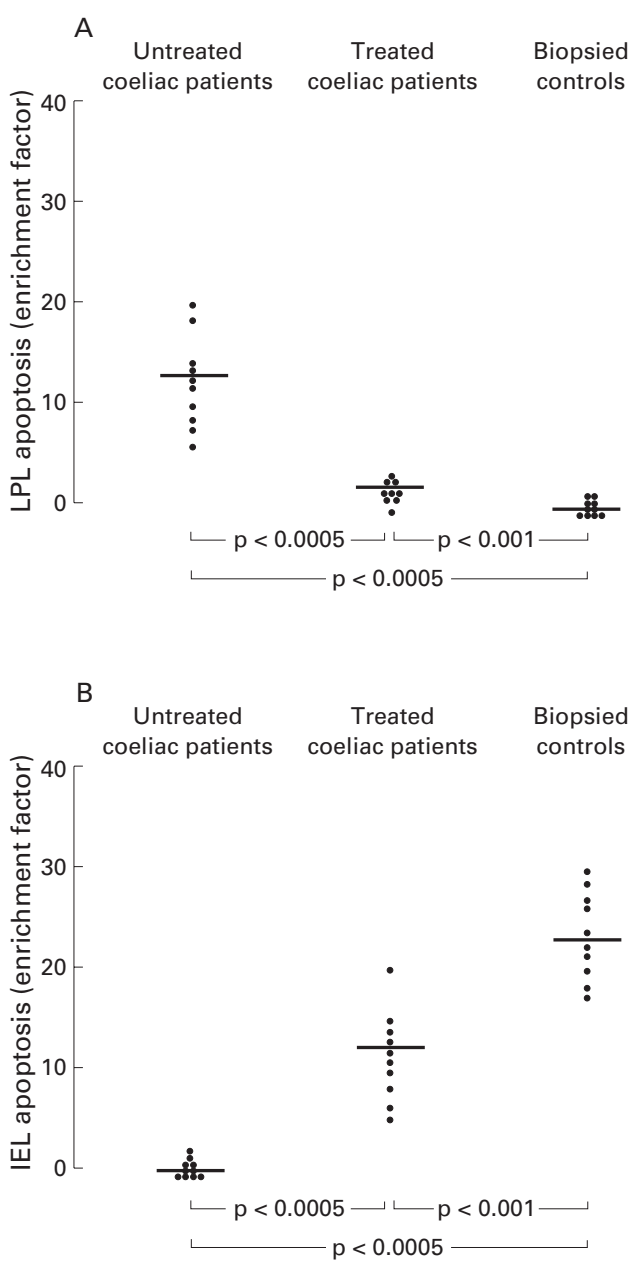

Figure 5 Detection of apoptosis on lamina propria lymphocytes (LPLs) (A) and intraepithelial lymphocytes (IELs) (B) by in vitro determination of cytoplasmic histone associated DNA fragments (mono and oligonucleosomes) in 10 untreated coeliac patients, 10 treated coeliac patients, and 10 biopsied controls.

Quantitative results are expressed as an enrichment factor of mono and oligonucleosomes compared with controls. Horizontal bars represent median values.

4. These data suggest that Fas/FasL binding may play a central role in determining enterocyte apoptosis in CD. On the other hand, when both control LPLs and IELs were cocultured with normal enterocytes, the proportions of $\mathrm{TUNEL}^{+}$target cells were poor (11.9 (2.5)\% and 12.7 (3.5)\%, respectively) and further decreased $(7.4(2.0) \%$ and $8.8(2.4) \%$, respectively) when ZB4 anti-Fas blocking was added to the coculture. The percentages of untreated coeliac TUNEL $^{+}$enterocytes, which were cultured without effector cells, did not significantly change in the absence $(31.1(5.2) \%)$ or presence $(28.9(4.0) \%)$ of ZB4 anti-Fas blocking antibody. The percentages of control TUNEL $^{+}$enterocytes, when cultured without effector cells, also did not significantly change in the absence $(6.1(1.3) \%)$ or presence (5.9 (1.4)\%) of ZB4 anti-Fas blocking antibody.

LPL AND IEL APOPTOSIS

Cell Death Detection ELISA for oligonucleosomes on cell lysates was used to evaluate apoptosis of freshly isolated LPLs and IELs. Figure 5A shows increased LPL apoptosis in untreated CD (median 13.4, range 6.9-20.8) compared with treated coeliac patients (median 2.5, range 0.9-4.2) and biopsied controls (median 0.6, range 0-1.8). In treated CD, LPL apoptosis was still significantly higher than in biopsied controls. Moreover, fig 6 shows that LPLs showed higher expression of Fas receptor in untreated CD (median 29.8\%, range 15.3-52.4 in 10 patients) compared with treated CD (median $4.9 \%$, range $0.2-14.0$ in 10 patients) and controls (median $2.7 \%$, range $0.1-1.7$ in 10 consenting subjects).

Figure 5B shows that the pattern of IEL apoptosis was opposite to that of LPLs. In untreated coeliac mucosa (median 0.2, range $0-2.1$ ), IEL apoptosis was lower than in treated coeliac patients (median 14.5, range 6.7-21.4) and biopsied controls (median 25.1, range 18.4-30.6). After treatment, IEL apoptosis was still significantly lower than in controls.

\section{Discussion}

In the present study, the cytotoxic activity of intestinal lymphocytes was quantified in CD. LPLs and IELs isolated from untreated coeliac mucosa shared higher levels of FasL and an increased and dose dependent killing activity against Fas expressing Jurkat cells compared with those isolated from treated coeliac and normal mucosa. In treated CD, both LPL and IEL cytotoxicity was still significantly higher than that of control lymphocytes. The lack of complete normalisation of cytotoxic activity by LPLs after a gluten free diet may be related to the capacity of dendritic cells to retain gliadin in its native form for a long time $\mathrm{e}^{15}$ and/or to the inadvertent ingestion of this protein. In fact, as regards the persistent activation of IELs, it has been shown that minimal amounts of gliadin may expand IEL populations and activate local immunity, even when the mucosal structure looks otherwise normal. ${ }^{16}{ }^{17}$ On the other hand,
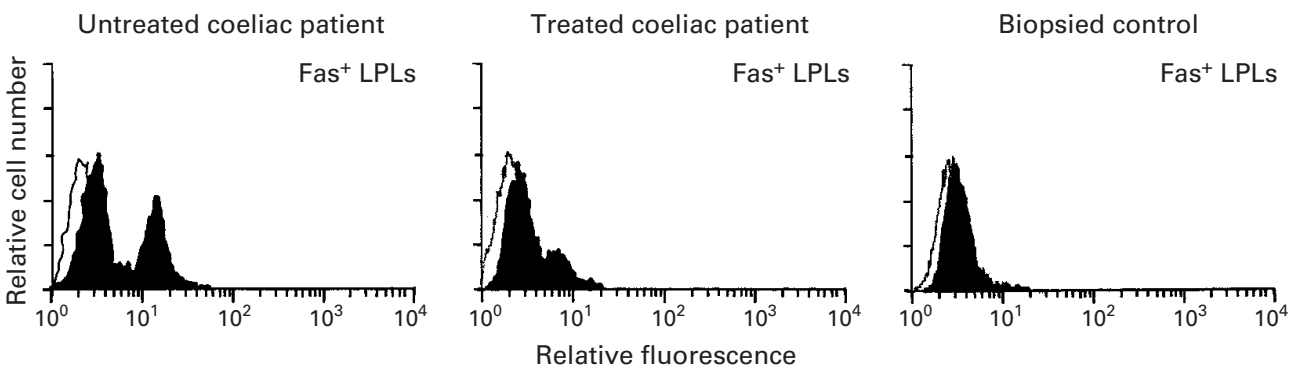

Figure 6 Representative flow cytometric histograms of Fas expression on lamina propria lymphocytes (LPLs) isolated from duodenal biopsy specimens of an untreated coeliac patient, a treated coeliac patient, and a biopsied control. 
our finding of higher FasL expression by both LPLs and IELs in those treated coeliac patients with persistence of mucosal lesions is further evidence of the involvement of FasL mediated lymphocyte cytotoxicity against epithelial cells in this condition.

To demonstrate that both LPLs and IELs function effectively as killers of enterocytes via a Fas/FasL interaction in active CD, the effect of the antagonist ZB4 anti-Fas antibody on apoptotic activity exerted by coeliac lymphocytes against enterocytes was analysed. The significant reduction in enterocyte apoptosis observed after coculturing coeliac lymphocytes and enterocytes in the presence of ZB4 antibody is additional proof of the involvement of the Fas/FasL system in CD associated enterocyte apoptosis. This evidence couples with our previous demonstrations that Fas is upregulated on coeliac enterocytes ${ }^{28}$ and because of the proximity of IELs and enterocytes, it is conceivable that the Fas/FasL mechanism may well be implicated in in vivo enterocyte apoptosis in this condition. With regard to LPLs, their killing activity may be exerted by membrane bound FasL through pores within the basement membrane, which allow their intimate contact with basolateral projections of enterocytes. ${ }^{18}$ In addition, other mechanisms may be operating, such as the role of a soluble form of FasL released by cleavage by matrix metalloproteases, ${ }^{19}$ which have been shown to be overexpressed in untreated coeliac mucosa. $^{20}$

As it has been shown that coeliac mucosa is a site of persistent $T$ cell activation and proliferation, ${ }^{41}$ it was therefore of interest to determine whether such cells themselves showed an abnormal pattern of apoptosis. LPLs shared an increased apoptosis and higher levels of Fas receptor in untreated CD compared with controls. It is known that Fas/FasL expression is closely related to the process of lymphocyte activation, and that their interaction is crucial for its control and termination. ${ }^{22}$ Therefore, it is likely that in untreated CD the increased propensity of LPLs to undergo apoptosis is a homeostatic mechanism consequent to their greater activation. ${ }^{23}$

A novel result of the present study is decreased apoptosis of IELs in untreated CD. Intestinal mucosa is chronically exposed to an abundance of dietary antigens and exogenous pathogens, and this continuous antigen challenge may abnormally activate IELs. ${ }^{24}{ }^{25}$ This activation does not normally result in inflammatory tissue damage, implying the involvement of an endogenous regulating mechanism. Our results in normal subjects confirm that a certain degree of lymphocyte apoptosis contributes to maintain IEL homeostasis by limiting expansion of activated $\mathrm{T}$ cells, and by ensuring the clearance of primed lymphocytes that are no longer required. ${ }^{26}$ Our finding of decreased IEL apoptosis is of particular relevance in the context of untreated CD. This defect, which is incompletely healed by a gluten free diet, may determine the nondeletion of autoreactive $\mathrm{T}$ cell clones, present in intestinally matured $\mathrm{T}$ cells, ${ }^{27}$ and/or expansion of IELs with an abnormal phenotype and a restricted repertoire, and this may precede the onset of a malignant condition. These findings are particular intriguing when considering that refractory sprue, which is an established complication of $\mathrm{CD}$, is characterised by an abnormal IEL population, ${ }^{28}$ and that intestinal $\mathrm{T}$ cell lymphoma arises from IELs. ${ }^{29}$

The possible mechanisms responsible for decreased IEL apoptosis in untreated CD are at present under investigation in our laboratory. Upregulation of Bcl-2 molecules ${ }^{30}$ and/or lack of downregulatory signals by coeliac enterocytes for IELs ${ }^{31}$ are likely candidates. In addition, IEL resistance to apoptosis may be a consequence of enterocyte secretion of interleukin 15 , a cytokine which has been shown to protect cells from induction of apoptosis ${ }^{32}$ and to be abundant in the epithelial compartment of active $\mathrm{CD}$ where it promotes the selective expansion of CD94 ${ }^{+}$IELs. ${ }^{33}$ Finally, in murine small intestine, IELs from villous tips and crypt portions show different propensities to undergo apoptosis. ${ }^{34}{ }^{35}$ On this basis, it would be interesting to analyse whether the abnormal spatial segregation of IELs in CD may contribute to their decreased apoptosis.

In conclusion, the present study demonstrates that coeliac LPLs and IELs show reversed patterns of apoptosis but a similar increase in FasL mediated cytotoxic activity. It is noteworthy that this activity, which has been fully documented for IELs, ${ }^{86}$ has also been reported for LPLs in a variety of disease conditions. ${ }^{37-39}$

1 Moss SF, Attia L, Scholes JV, et al. Increased small intestinal apoptosis in coeliac disease. Gut 1996;39:811-17.

2 Ciccocioppo R, Di Sabatino A, Parroni R, et al. Increased enterocyte apoptosis and Fas/FasL system in celiac disease. Am $\mathcal{F}$ Clin Pathol 2001;115:494-503.

3 Marsh MN, Crowe PT. Morphology of the mucosal lesion in gluten sensitivity. Baillières Clin Gastroenterol 1995;9: 273-327.

4 Halstensen TS, Brandtzaeg P. Activated T lymphocytes in the coeliac lesions: non-proliferative activation (CD25) of $\mathrm{CD} 4^{+} \alpha / \beta$ cells in the lamina propria but proliferation (Ki$67)$ of $\alpha / \beta$ and $\gamma / \delta$ cells in the epithelium. Eur f Immunol 1993) of $\alpha / \beta$ and $\gamma / \delta$.

5 MacDonald TT, Spencer J. Evidence that activated mucosal $T$ cells play a role in the pathogenesis of enteropathy in human small intestine. F Exp Med 1988;167:1341-9.

6 Sakai T, Kimura Y, Inagaki-Ohara K, et al. Fas-mediated cytotoxicity by intestinal intraepithelial lymphocytes during acute graft-versus-host disease in mice. Gastroenterology 1997;113:168-74.

7 Lin T, Brunner T, Tietz B, et al. Fas ligand-mediated killing by intestinal intraepithelial lymphocytes. Participation in intestinal graft-versus-host disease. $\mathcal{F}$ Clin Invest 1998;101: $570-7$.

8 Ciccocioppo R, Di Sabatino A, Parroni R, et al. Cytolytic mechanisms of intraepithelial lymphocytes in coeliac disease. Clin Exp Immunol 2000;120:235-40.

9 Moretta A. Molecular mechanisms in cell-mediated cytotoxicity. Cell 1997;90:13-18.

10 Akbar AN, Borthwick N, Salmon M, et al. The significance of low Bcl-2 expression by CD45RO $\mathrm{T}$ cells in normal individuals and patients with acute viral infections: the role of apoptosis in T cell memory. F Exp Med 1993;178:42738.

11 Kabelitz D, Pohl T, Pechhold K. Activation induced cell death (apoptosis) of mature peripheral $\mathrm{T}$ lymphocytes. Immunol Today 1993;14:338-9.

12 Matzinger P. The JAM test. A simple assay for DNA fragmentation and cell death. F Immunol Methods 1991;145: 185-92.

13 Dunnil MS, Whitehead R. A method for the quantitation of small intestinal biopsy specimens. F Clin Pathol 1972;25: 243-6.

14 Corazza GR, Frazzoni M, Dixon MF, et al. Quantitative assessment of the mucosal architecture of jejunal biopsy specimens: a comparison between linear measurement, stereology, and computer aided microscopy. $\mathcal{F}$ Clin Pathol 1985;38:765-70. 
15 Tew JG, Phipps RP, Mandel TE. The maintenance and regulation of the humoral immune response: persisting antigen and the role of follicular antigen-binding dend

16 Catassi C, Rossini M, Rätsch IM, et al. Dose dependent effects of protracted ingestion of small amounts of gliadin in coeliac disease children: a clinical and jejunal morphometric study. Gut 1993;34:1515-19.

17 Mayer M, Greco L, Troncone R, et al. Compliance of adolescents with coeliac disease with a gluten-free diet. Gut 1991;32:881-5.

18 Hershberg RM, Mayer LF. Antigen processing and presentation by intestinal epithelial cells-polarity and complexity. Immunol Today 2000:21:123-8.

19 Kayagaki N, Kawasaki A, Ebata T, et al. Metalloproteinasemediated release of human Fas ligand. $\mathcal{f}$ Exp Med 1995;182:1777-83.

20 Daum S, Bauer U, Foss HD, et al. Increased expression of mRNA for matrix metalloproteinases- 1 and -3 and tissue inhibitor of metalloproteinases- 1 in intestinal biopsy speciinhibitor of metalloproteinases-1 in intestinal biopsy specimen

21 Nilsen EM, Lundin KE, Krajci P, et al. Gluten specific, HLA-DQ restricted T cells from coeliac mucosa produce cytokines with Th1 or Th0 profile dominated by interferon- $\gamma$. Gut 1995;37:766-76.

22 Dhein J, Walczak H, Baumler C, et al. Autocrine T-cell suicide mediated by APO-1 (Fas/CD95). Nature 1995;373 438-41.

23 Boirivant M, Pica R, De Maria R, et al. Stimulated human lamina propria $\mathrm{T}$ cells manifest enhanced Fas-mediated apoptosis. $\mathcal{F}$ Clin Invest 1996;98:2616-22.

24 Janeway CA, Jones BJ, Hayday A. Specificity and function of $\mathrm{T}$ cells bearing $\gamma \delta$ receptor. Immunol Today 1988;9:73-6.

25 Evert EC. Do the CD45 $\mathrm{RO}^{+} \mathrm{CD}^{+}$intestinal intraepithelial T lymphocytes have the characteristic of memory cells? Cell Immunol 1993;147:331-40.

26 Morimoto Y, Hizuta A, Ding EX, et al. Functional expression of Fas and Fas ligand on human intestinal intraepithelial lymphocytes. Clin Exp Immunol 1999;116: intraep.

27 Rocha B, von Boehmer H, Guy-Grand D. Selection of intraepithelial lymphocytes with $\mathrm{CD} 8 \alpha / \alpha$ co-receptors by self-antigen in the murine gut. Proc Natl Acad Sci USA 1992;89:5336-9

28 Cellier C, Patey N, Mauvieux L, et al. Abnormal intestinal intraepithelial lymphocytes in refractory sprue. Gastroenterology 1998;114:471-81.
29 Spencer J, Cerf-Bensussan N, Jarry A, et al. Enteropathyassociated $\mathrm{T}$ cell lymphoma is recognized by a monoclonal ntibody (HML-1) that defines a membrane molecule on human mucosal lymphocytes. Am F Pathol 1988;132:1-5.

30 Strasser A, Harris AW, Huang DC, et al. Bcl-2 and Fas/APO-1 regulate distinct pathways to lymphocyte apoptosis. EMBO ₹ 1995;14:6136-47.

31 Yamamoto M, Fujihashi K, Kawabata K, et al. A mucosal intranet: intestinal epithelial cells down-regulate intraepithelial, but not peripheral, $\mathrm{T}$ lymphocytes. I Immunol 1998;160:2188-96.

32 Bulfone-Paus S, Ungureanu D, Pohl T, et al. Interleukin-15 protects from lethal apoptosis in vivo. Nat Med 1997;3: $1124-9$

33 Jabri B, Patey-Mariaud De Serre N, Cellier C, et al. Selective expansion of intraepithelial lymphocytes expressing the HLA-E-specific natural killer receptor CD94 in celiac disease. Gastroenterology 2000;118:867-79.

34 Yamamoto M, Fujihashi K, Amano M, et al. Cytokine synthesis and apoptosis by intestinal intraepithelial ymphocytes: signaling of high density $\alpha \beta \mathrm{T}$ cell receptor ${ }^{+}$ and $\gamma \delta \mathrm{T}$ cell receptor ${ }^{+} \mathrm{T}$ cells receptor-CD3 complex results in interferon- $\gamma$ and interleukin- 5 production, while low density $\mathrm{T}$ cells undergo DNA fragmentation. Eur $\mathcal{F}$ Immunol 1994;24:1301-6.

35 Kawabata S, Boyaka PN, Coste M, et al. Intraepitelial lymphocytes from villus tip and crypt portions of the murine mall intestine show distint characteristics. Gastroenterology 1998;115:866-73.

36 Gelfanov V, Gelfanova V, Lai YG, et al. Activated $\alpha \beta-C D 8^{+}$, but not $\alpha \alpha-\mathrm{CD}^{+}$, TCR- $\alpha \beta^{+}$murine intestinal intraepithelial lymphocytes can mediate perforin-based cytotoxicity, whereas both subsets are active in Fas-based cytotoxicity. $\mathcal{F}$ Immunol 1996;156:35-41.

37 Ueyama H, Kiyohara T, Sawada N, et al. High Fas ligand expression on lymphocytes in lesions of ulcerative colitis. Gut 1998;43:48-55.

38 Nishio A, Katakai T, Oshima C, et al. A possible involvement of Fas-Fas ligand signaling in the pathogenesis of murine autoimmune gastritis. Gastroenterology 1996;111: $959-67$.

39 Simpson SJ, De Jong YP, Shah SA, et al. Consequences of Fas-ligand and perforin expression by colon $\mathrm{T}$ cells in a mouse model of inflammatory bowel disease. Gastroenterology 1998;115:849-55. 